
\title{
28 Research Square \\ A Ten-Year Retrospective Study of Invasive Candidiasis in a Tertiary Hospital
}

\section{Zhihui Yang ( $\nabla$ yangzhihui0508@sina.com )}

Peking University First Hospital https://orcid.org/0000-0003-1193-6894

\section{Yinggai Song}

Peking University First Hospital

\section{Ruoyu Li}

Peking University First Hospital

\section{Research Article}

Keywords: invasive candidiasis, epidemiology, risk factors, antifungal resistance, treatment, mortality.

Posted Date: May 6th, 2021

DOI: https://doi.org/10.21203/rs.3.rs-321128/v1

License: (1) This work is licensed under a Creative Commons Attribution 4.0 International License. Read Full License 


\section{Abstract}

Background: Invasive candidiasis (IC) is a leading cause of mycosis-associated mortality worldwide. Delay in diagnosis and antifungal therapy significantly increases the risk of death. Thus, comprehensive knowledge of IC is required to recognize the IC onset timely.

Objectives: This study aimed to evaluate the epidemiological, clinical and mycological characteristics of IC in China.

Methods: A ten-year retrospective study including 183 IC episodes was conducted in a tertiary hospital in Beijing, China.

Results: The overall incidence rate during 2010-2019 was 0.261 episodes per 1,000 discharges. Candidemia (71.0\%) was the major infective pattern. $70.3 \%$ of the patients tested positive for Candida spp. colonization before IC and the median time to develop an invasive infection after colonization was 13.5 days (interquartile range: 4.5-37.0 days). Candida albicans (45.8\%) was the most prevalent species, followed by Candida parapsilosis (19.5\%), Candida glabrata (14.2\%) and Candida tropicalis (13.7\%). C. non-albicans IC was more common in patients with severe anemia $(p=0.018)$, long-term hospitalization $(p=0.015)$, hematologic malignancies $(p=0.002)$, continuous administration of broad-spectrum antibiotics $(p<0.001)$ and mechanical ventilation $(p=0.012)$. In vitro resistance testing showed that $14.4 \%$ of the Candida isolates were resistant/ non-wild type (non-WT) to fluconazole, followed by voriconazole (9.6\%), micafungin (3.8\%) and caspofungin (2.9\%). Fluconazole was the most commonly used drug to initiate antifungal therapy both before and after the proven diagnosis ( $52.6 \%$ and $54.6 \%$, respectively). 30 -day and 90 -day all-cause mortality was $24.5 \%$ and $32.7 \%$, respectively.

Conclusions: The incidence of IC was lower in recent years. C. non-albicans contributed to more than half of the IC cases. Fluconazole could still be used as first-line therapy if its resistance/ non-WT was not prevalent. The mortality of IC was still high. A prospective, multi-center surveillance to clinical and mycological characteristics of IC is required.

\section{Introduction}

Invasive candidiasis (IC) refers to infections by Candida spp. in the bloodstream (AKA candidemia) or other deep sites of the human body including the abdominal cavity, bone, brain, eye, heart, kidney, lung, liver and spleen [1]. IC is a leading cause of mycosis-associated mortality with all-cause mortality of 46$75 \%$ and attributable mortality of $10-49 \%$ [1-6]. Several hospital-based studies have reported an increase in the IC incidence since 2000s, which requires clinicians to be alert [7-9].

Currently, a proven diagnosis of IC still relies on routine fungal cultures, which have low sensitivity ranging from $21 \%$ to $71 \%$ and may take $2-3$ days averagely to detect the infection [1]. However, early diagnosis and antifungal therapy are essential as empirical treatment within 24 hours of a septic shock due to candidemia could effectively reduce the mortality from $97.6 \%$ to $52.8 \%$ [10]. Thus, a 
comprehensive grasp of IC is important, especially for identifying the vulnerable population and applying appropriate empirical treatment, as there are no specific clinical symptoms or signs for IC. However, previous studies mainly focused on either mycological aspects or clinical risk factors of candidemia. Few studies have combined the clinical and mycological aspects and comprehensively summarized both candidemia and deep-seated candidiasis. Furthermore, from the mycological aspect, the distribution of pathogens responsible for IC is ever changing, as well as the distribution of drug resistance. Though Candida albicans remains to be the most common species worldwide, accounting for approximately $37.2-61.0 \%$ of the IC cases $[4,7,8,11-13]$, the Candida non-albicans caused IC became increasingly common in the recent decades $[4,14]$, which resulted in increasing resistance in azoles. Thus, there is the need to comprehensively summarize the IC characteristics and continuously update the mycological characteristics of IC with latest data.

This study aimed to combine the latest mycological and clinical data of IC cases in a tertiary hospital in Beijing, in order to comprehensively investigate the incidence, population distribution, risk factors and the status of diagnosis and treatment of IC in China.

\section{Subjects And Methods}

\subsection{Hospital setting and Study population selection}

This retrospective study was conducted in Peking University First Hospital, which is a "rank A tertiary" hospital with 64 wards and more than 90,000 admissions per year. All the yeast isolates (506 isolates in total) extracted from bloodstream and deep viscera from January 2010 to December 2019 were preserved at the Research Center for Medical Mycology of this hospital. 212 isolates from 183 proven diagnosed IC episodes with comprehensive hospitalized medical records were included in this study (Fig. 1). Proven diagnosis of IC was made according to the revised and updated consensus definitions of invasive fungal disease by yeasts published by EORTC/MSG[1] in 2019 [15]. Patients were included if Candida spp. were recovered from sterile materials, including blood, central venous catheter (CVC) tips, cerebrospinal fluid (CSF), hydrothorax, ascites, synovial fluid, sterile tissue (e.g. bone), and freshly placed (within 24 hours) drains which included abdominal drainage fluid, pleural drainage fluid, abscess drainage and bile in this study.

Two positive culture results by an identical Candida species within one month or by different Candida species within two weeks were considered as one episode of infection.

\subsection{Data collection}

For each proven IC episode, patient data was collected including demographic information, hospitalized wards, underlying diseases, other potential risk factors for IC, time from admission to IC onset, time from Candida colonization to onset, infected sites and Candida species, (1,3)- $\beta$-D-Glucan results, antifungal resistance, antifungal therapies, and time from onset to death. 


\subsection{Definitions}

All the potential risk factors for IC existed within 30 days before onset were recorded. Prolonged use of corticosteroids was defined as a mean minimum dose of $0.3 \mathrm{mg} / \mathrm{kg} /$ day of prednisone equivalent for at least 3 weeks. Broad-spectrum antibiotics was defined as antibiotics covering both Gram-positive and Gram-negative bacteria, mainly including the third and fourth-generation cephalosporins, carbapenems and quinolones. Exposure to broad-spectrum antibiotics was defined as the use of these drugs within 2 weeks preceding IC onset, while long-term exposure was defined as exposure to these antibiotics for more than 3 weeks continuously. Neutropenia was defined as the absolute neutrophil count lower than 500 cells/ $\mu \mathrm{L}$. Candida colonization was defined as the isolation of a Candida species from at least one unsterile site, including sputum, bronchoalveolar lavage fluid (BALF) or tracheal secretions, gastric fluid, urine, stool, swab from oropharynx, wound, perinea, or catheter insertion sites, and drained fluids collected from catheters placed more than 24 hours [16]. As colonization was only monitored in patients with clinical symptoms, the colonization would be considered as none if the test was not conducted. 'Candida Score' wad defined as the sum of severe sepsis (2 points), surgery (1 point), total parenteral nutrition (TPN, 1 point) and multifocal colonization (i.e. colonization at more than one site, 1 point). Patients with a Candida score $\geq 3$ are prone to IC $[17,18]$.

\subsection{Species identification and antifungal susceptibility testing}

All the included isolates were re-identified to the species level by MALDI-TOF-MS using the MALDI Biotyper RTC 4.0 software (Bruker Daltonik). Antifungal susceptibility tests were performed using an SYO Y010 panel tray (Thermo Scientific, Cleveland, $\mathrm{OH}$, United States). Minimum inhibitory concentration (MIC) data were determined using the CLSI[2]-approved standard M27-S4, and the isolate susceptibility was interpreted mainly according to the updated species-specific CLSI clinical breakpoints (CBPS) or epidemiological cutoff values (ECVs), as described in the study of Song et al.[19].

\subsection{Statistical analysis}

A descriptive analysis of baseline characteristics was conducted. Categorical baseline characteristics of the patients were presented by number (percentage) and compared using chi-squared tests. Normality of the continuous variables was checked using the Shapiro-Wilk test. Non-normally distributed continuous baseline characteristics were presented by median values (interquartile range, IQR) and compared using Mann-Whitney $\mathrm{U}$ tests, while normally distributed continuous characteristics were presented by mean values ( \pm standard deviation) and compared using t-tests. All tests of significance were two-tailed.

Univariable and multivariable logistic regression models were used when determining the risk factors influencing 90-day all-cause mortality. A complete-case analysis was employed as the clinical outcomes 
of 24 episodes were missing. The multivariable logistic regression models adjusted for variables that were both clinically considered as confounding factors and showed possible association with 90-day mortality in the univariable regression $(p<0.100)$ in the meanwhile.

All data analysis was conducted using STATA/IC 15 [20]. A p-value lower than 0.050 was considered as statistically significant.

\section{Results}

\subsection{Incidence and patient demographics}

The overall IC incidence rate in this comprehensive hospital during 2010-2019 was 0.261 episodes per 1,000 discharges or 0.032 episodes per 1,000 patient*days. The incidence of IC ranged from 0.081 per 1,000 discharges (in 2017) to 0.432 per 1,000 discharges (in 2012). The incidence decreased in the recent five years compared to the first five years (Fig. 2).

Among the included 183 episodes of IC, $67.8 \%$ (124/183) were male and 32.2\% (59/183) were female. The median age was 66 years old (IQR: $40-78$ years old, range: 0 days to 96 years old). The majority of patients were $\geq 65$ years old $(54.1 \%, 99 / 183)$ while 22 patients $(12.0 \%)$ were $\leq 1$ year old. 130 episodes were diagnosed as candidemia, 50 were non-candidemic invasive candidiasis, and three were multiseated infection with candidemia (Table 1). The infected sites varied among different age groups (Table 1). Blood/CVC was the dominant site of infection for all the age groups. Central nervous system candidiasis mainly affected infants ( 1 month - 1 year old, $p<0.001)$ while infection in other fluids (except for CSF) and drains mainly occurred in elderly adults (Table 1). Mortality rate was higher in aged patients $\geq 65$ years old $(37.4 \%, p=0.080)$. $43.2 \%$ (79/183) of the IC episodes occurred in intensive care units (ICUs), $30 \%(53 / 183)$ in surgical wards, $23.0 \%(42 / 183)$ in medical wards and $4.9 \%(9 / 183)$ in pediatric wards (Fig. 3). In addition, surgical ICU was the most affected ward among all the ICUs, followed by respiratory ICU. The comprehensive ward, where most patients had a long period of hospitalization, was the most affected ward by IC among all the medical wards.

\subsection{Risk factors}

Common host factors of IC patients included central venous catheterization $(62.1 \%, 108 / 174)$, age $\geq 65$ years old $(56.9 \%, 99 / 174)$, organ failure $(56.9 \%, 99 / 174)$, other deep-seated bacterial infection $(48.3 \%$, $84 / 174)$, major surgery $(46.6 \%, 81 / 174)$, long-term use of broad-spectrum antibiotics $(42.0 \%, 73 / 174)$, TPN $(41.4 \%, 72 / 174)$, and ICU hospitalization $(40.2 \%, 70 / 174)$ (Table 2$)$.

Specifically, the hemoglobin was $9.6 \mathrm{~g} / \mathrm{L}$ lower, the hospitalization was 21.5 days longer and the average Candida Score was 0.6 points higher averagely in the candidemia group compared to the non-candidemic 
IC group ( $p=0.011, p<0.001$ and $p<0.001$ respectively). In the candidemia group, there were significantly more patients with hematologic malignancies $(p=0.045)$, neutropenia $(p=0.023)$, respiratory failure $(p<0.001)$, other deep-seated bacterial infection $(p=0.017)$, hemodialysis $(p=0.013)$, long-term use of broad-spectrum antibiotics $(p<0.001), C V C(p<0.001)$, mechanical ventilation $(p=0.001)$, sepsis $(p=0.001)$ and TPN $(p=0.003)$. In contrast, there were significantly more patients with mould infection $(p=0.039)$, cholecystitis $(p=0.025)$, ileus $(p=0.025)$, digestive tract perforation $(p<0.001)$ and gastrointestinal surgery history $(p=0.042)$ in the non-candidemic IC group.

Differently, for neonates, the most common host factors were low birth weight (100\%), maternal late pregnancy $(100 \%)$, premature $(88.9 \%, 8 / 9)$, administration of CVC $(100 \%)$ and mechanical ventilation $(88.9 \%, 8 / 9)$ (Supplementary Table 1).

Candida colonization was another common risk factor for acquiring IC. 74/183 patients had tested for Candida colonization before developing IC. 70.3\% (52/74) of the patients were tested positive for colonization. $45.9 \%$ (34/74) of the patients had Candida spp. colonized at one unsterile site and $24.3 \%$ $(18 / 74)$ of the patients had multifocal colonization ( 14 at 2 sites, 3 at 3 sites and 1 at 4 sites). Among the 52 patients with Candida colonization before IC, the median time to develop an invasive infection after the colonization was 13.5 (IQR: 4.5-37, range: 1-255) days. Sputum/BALF/tracheal secretions was the most frequent site with Candida colonization (Supplementary Table 2). Candidemia was more often in patients with colonization in urine $(p=0.018)$ while infection in drains was more often in patients with colonization in sputum ( $p=0.032)$. Notably, 17 patients acquired IC by a Candida species different from the colonized ones. Among them, five patients who were colonized with C.albicans developed C.glabrata invasive infection afterwards. In addition, five patients had colonization with multiple Candida species before the onset of IC by a single Candida species.

\subsection{The fungi distribution}

C.albicans was the most prevalent species, contributing to $45.8 \%$ of the cases, followed by C.parapsilosis (19.5\%), C.glabrata (14.2\%), C.tropicalis (13.7\%) and C.krusei (3.7\%) (Fig. 4). Overall, the cases caused by C.albicans was similar to $C$. non-albicans except for the year 2012 and 2017. However, the variety of the infected species increased in 2019. C.krusei also became more frequent in the recent five years (Fig. 5).

When comparing cases caused by $C$. albicans and $C$. non-albicans, $C$. non-albicans was more likely to cause candidemia $(p=0.002)$ while $C$. albicans was more likely to infect CSF $(p=0.002)$ and drains $(p=0.022)$. Patients with $C$. non-albicans $I C$ had a hemoglobin $6.4 \mathrm{~g} / \mathrm{L}$ lower averagely $(p=0.034)$ and had stayed in the hospital for a median of 8 days longer $(p=0.011)$ than those with $C$. albicans IC. In addition, C.non-albicans IC had a preference in patients with severe anemia $(p=0.018)$, long-term hospitalization $(p=0.015)$, hematologic malignancies $(p=0.002)$, administration of broad-spectrum antibiotics continuously $(p<0.001)$, mechanical ventilation $(p=0.012)$, previous exposure to fluconazole $(p<0.001)$ and voriconazole $(p=0.001)$. In contrast, $C$. albicans tended to infect patients with solid-organ malignancies $(p=0.001)$ and those undergoing surgeries $(p=0.003)$. There was no significant difference 
observed between two groups regarding other risk factors listed in Table 3. Associations between basic medical conditions and other Candida spp. caused invasive infection were listed in Supplementary Table 3.

\subsection{Diagnostic methods}

All the patients included in the study were diagnosed by culture according to the study design. Time used to detect the fungal colony growth was reported to be 28.0 (IQR: 14.5-38.5) hours in 68 blood sample.

1-3- $\beta$-D-glucan (BDG) test was performed in 110 episodes of IC within seven days before and after the date when proven diagnosis was made according to culture. Only $33(30 \%)$ were tested positive for BDG. The cut-off for a positive result in this center was $100 \mathrm{pg} / \mathrm{ml}$. However, the median value of BDG within seven days of proven diagnosis was 30.5 (IQR 5-197) pg/ml.

Among those with a positive 1-3- $\beta-D-g l u c a n$, the median time for BDG to rise positive after proven diagnosis according to culture was 3 (IQR: 0-12) days. Probable diagnosis was made in ten episodes according to a positive BDG result before a successful recovery of a yeast.

\subsection{Antifungal resistance}

The drug resistance of the included 212 isolates are summarized in Supplementary Table 4. In vitro resistance testing showed that $14.4 \%(30 / 208)$ of Candida isolates were resistant/ non-wild type (nonWT) to fluconazole, 9.6\% (20/208) were resistant /non-WT to voriconazole, 3.8\% (8/208) were resistant to micafungin, 2.9\% (6/208) were resistant to caspofungin, while all of the Candida isolates were wild type (WT) to Amphotericin B. Fluconazole resistance was significantly more prevalent in C.tropicalis $(37.0 \%$, $\mathrm{p}<0.001)$ than other species, and voriconazole non-WT phenotype was more prevalent in C.glabrata $(58.6 \%, p<0.001)$.

59 isolates belonged to patients who had previously exposed to fluconazole with a median of 12 (IQR: 626) days before isolation. For the total 212 isolates, the fluconazole-resistant isolates had longer duration of the previous exposure to fluconazole than the fluconazole non-resistant isolates (median/ 10-90\% quartile range: $0 / 0-82.5$ days vs 0/0-16 days, $p=0.052$ ). Furthermore, resistance to voriconazole was significantly associated with longer previous exposure to fluconazole $(p=0.002)$, and resistance to fluconazole was significantly associated with longer previous exposure to voriconazole as well $(p<0.001)$. Similarly, resistance to micafungin might also be associated with longer previous exposure to caspofungin ( $\mathrm{p}=0.053)$. Further tests confirmed that resistance to fluconazole and voriconazole were mutually associated $(p<0.001)$, and resistance to micafungin and caspofungin were also mutually associated $(p<0.001)$.

\subsection{Treatment}


The median time taken to administrate systematic antifungal drugs was 1 day (IQR: -1 to 3 days) after proven diagnosis. Prophylaxis/ empirical/ preemptive antifungal drugs were given to 38 patients (20.8\%) and targeted antifungal drugs were given to 152 patients (83.1\%). The most commonly used drug to initiate antifungal therapy both before and after proven diagnosis was fluconazole ( $52.6 \%$ and $54.6 \%$, respectively). Though the main antifungal therapy changed (including the dosage and type of drugs) from the initial ones in 71 cases, the main antifungal drugs used during the disease course was still fluconazole (41.2\%) (Table 4). Compared with micafungin, initiating antifungal therapy with fluconazole had better prognosis ( $p=0.031$, Supplementary Table 5 ). No statistically significant differences in mortality were found among the mainly used antifungal drugs during the disease course (Supplementary Table 5). To note, there were 31 patients did not receive any antifungal drugs and 64.5\% (20/31) of them survived after removing the catheters, effective drainage or surgery (details in Supplementary Table 6). In this study, neither prophylaxis antifungal treatment in hematologic malignancies nor empirical/ preemptive antifungal treatment in all population was associated with better survival $(p=0.090$ and 0.170 , respectively).

\subsection{Prognosis}

24 patients requested a discharge despite a poor prognosis. Among the 159 left infection episodes, there were 52 deaths $(32.7 \%)$ within 90 days of diagnosis and 38 deaths $(23.9 \%)$ were due to IC. 7 -day and 30 day all-cause mortality was $10.1 \%$ (16/159) and 24.5\% (39/159), respectively, and 7-day and 30-day attributable mortality was $8.2 \%(13 / 159)$ and $18.9 \%(30 / 159)$, respectively. The median time to attributable mortality after proven diagnosis was 14.0 (IQR: 4.5-34.0, range: 1.0-143.0) days.

More deaths were found in patients infected with C.glabrata $(45.8 \%, p=0.137$, Table 3$)$. In the univariable logistic regression, more deaths occurred in patients with increased ages (Odds ratio [OR] 1.02, $p<0.001$ ), organ failure (OR 8.86, $p<0.001)$, especially heart failure (OR 4.63, $p<0.001)$, renal failure (OR 4.54, $p<0.001)$ and respiratory failure (OR 6.90, $p<0.001)$, bacterial infection in other sites (OR 2.13, $p=0.009)$, ICU hospitalization (OR 2.02, p=0.039), administration of broad-spectrum antibiotics continuously (OR $3.43, p<0.001)$ and mechanical ventilation (OR 3.05, $p=0.002)$, previous exposure to fluconazole (OR 2.49, $\mathrm{p}=0.028)$, and higher Candida Score (OR 1.44, $\mathrm{p}=0.013$ ) especially with sepsis (OR 2.67, $\mathrm{p}=0.011)$. After adjusting for confounding factors, risk factors that could significantly increase the risk of 90-day allcause mortality included hematologic malignancies $(O R=44.29, p=0.011)$, neutropenia $(O R=38.31$, $p=0.014)$, heart failure $(O R=3.62, p=0.025)$, respiratory failure $(O R=7.13, p=0.003)$, digestive tract perforation ( $O R=13.02, p=0.022)$, administration of broad-spectrum antibiotics continuously $(O R=5.30$, $p=0.008)$, and TPN $(O R=3.30, p=0.023)$. In contrast, protective factors against mortality included longterm hospitalization $(\mathrm{OR}=0.12, \mathrm{p}=0.006)$ and $\mathrm{CVC}$ induced IC $(\mathrm{OR}=0.12, \mathrm{p}=0.006)$ (Supplementary Table $5)$.

\section{Discussion}


This was a comprehensively retrospective study including 183 invasive candidiasis cases according to the latest diagnostic guideline by EORTC/MSG [15]. Descriptive analysis, chi-squared tests, Mann-Whitney $\mathrm{U}$ tests and t-tests were used to describe the characteristics of the included patients and Candida species, antifungal susceptibility, status of diagnostic tests, treatment and prognosis. Logistic regression models were employed to investigate the association between risk factors/ treatment and prognosis.

The incidence of IC in this study (0.26\%) was relatively lower compared to the incidence in previous studies based on hospital settings, which was reported to be $1.9-2.4 \%$ in the US, $1.18 \%$ o in Latin America, 0.92-1.19\%o in Europe and 0.13-1.22\%o in Asia [7, 12]. In addition, though the incidence of IC was increasing in several centers owing to the increasingly intensive care such as long-term administration of broad-spectrum antibiotics, the ten-year period in this study witnessed an overall decrease in the IC incidence. These might be due to the increasing awareness in this disease and the effective prevention of nosocomial infection. Several studies in Asia also reported a decrease in healthcare-associated infection including nosocomial candidemia owing to sequential multifaceted interventions such as hand hygiene, care bundles, and antimicrobial stewardships in the recent years [6, $13,21]$. In addition, $43.2 \%$ (79/183) of the episodes in this study occurred in ICU settings, similar to the global statistics, which was approximately $44.5-50.0 \%[1,22-24]$.

Since the proven diagnose of IC based on culture takes a median of 2-3 days and has low sensitivity of $21-71 \%$ [1], timely empirical therapy is often required to reduce mortality. In order to predict the possible infected Candida species and timely prescribe empirical antifungal drugs targeting the suspected species, grasping the geographical distribution and clinical characteristics of each Candida species is essential. In this study, C.albicans was the most common species (45.8\%) of the isolates, followed by C.parapsilosis (19.5\%). This is consistent with many studies in China, including the multicenter China-SCAN study[1] and CHIF-NET[2] study, which all reported C.parapsilosis (19.8-27.8\%) to be the second common species following C.albicans $[8,11,13,25,26]$. There were also single-center studies in China reporting C.tropicalis (18.0\%) and C. glabrata (43.0\%) as the most popular C.non-albicans [6, 7]. Grasping the local prevalence is essential as the Candida spp. distribution varies from center to center. In terms of clinical prevalence, this study found that $C$. non-albicans was more often to cause bloodstream infection than infections in other deep sites, which was consistent with previous findings [25,27]. Specifically, the present study and several previous studies all found that $C$. parapsilosis had a preference in younger patients without neutropenia or immunosuppressed condition, while C.glabrata and C. krusei had a preference in aged patients with poorer conditions such as severe anemia, renal disease and ventilator dependence $[4,27]$. C.tropicalis and $C$. krusei were additionally found to be associated with hematologic malignancies and immunosuppression in this study and the study of Horn et al.[27]. Previous exposure to antifungal drugs may also influence the infected species. Prior fluconazole therapy was found to be a risk factor for infection with fluconazole non-susceptible $C$.non-albicans [7]. This study further found an association between C.tropicalis and prior exposure to voriconazole, and between C.glabrata and prior exposure to fluconazole. $C$. krusei candidemia was also reported to be associated with prior use of antifungal agents [27]. All this information is essential, including local Candida spp. prevalence 
distribution, the infected sites and medical conditions of the patients, since they can provide clues for the infected Candida species and help clinicians to make targeted prescription timely.

In terms of risk factors, abnormal colonization is regarded as the prerequisite of the subsequent invasive candidiasis. $70.3 \%$ of the patients in this study had Candida colonization before IC onset. The median time to develop IC after colonization was 13.5 days in this study and reported to be 7-25 days in nonneutropenic ICU patients $[16,28]$. Thus, additional attention should be paid to those with abnormal Candida colonization, especially to those with colonization in more than two sites, heavy colonization in more than one site, and colonization in the urine, who have significantly higher risk of IC [28]. Interestingly, in this study, $23.0 \%$ of the patients developed IC by a Candida species different from the colonized Candida species and $24.3 \%$ colonized with multi-Candida species. This phenomenon might be explained as the colonization with different Candida species contributed to the burden of fungi, which eventually caused symptomatic infections [16]. Thus, when choosing antifungal drugs for the suspected infected species in the empirical treatment, the clinicians should be aware that the colonized Candida species may be different from the ones causing invasive infection. In addition, as Candida is a common symbiotic yeast, the positive predictive value of colonization for IC is low (2-4\%), whereas the negative predictive value is high (99-100\%) [28]. Dynamic monitoring of colonization can be laboratory intensive and significantly increase the economic burden of patients [29]. In this circumstance, Candida Score is an alternative to predict the risk of IC development, as patients with a Candida Score $\geq 3$ are prone to IC (sensitivity $81 \%$, specificity $74 \%$ ) and would benefit from empirical treatment $[17,18]$. This study also found that patients with higher Candida Score had increased risk of mortality, thus should be paid more attention.

Culture from blood or sterile sties is currently the gold standard for proven diagnosis. Though a successful recovery of Candida spp. might take 2-3 days [1], detecting fungal growth in blood sample culture just took a median of 28 hours in this study. Still, empirical treatment should be delivered when waiting for the results of culture if IC is suspected, since timely antifungal therapy within 24 hours can significantly reduce the mortality of candidemia [10].

Serum 1,3- $\beta$-D-glucan (BDG) test is another widely used test to facilitate diagnosis. $\beta$-D-glucan is a panmarker for various fungal infections. Previous studies have demonstrated its high sensitivity $(64.8 \%-89 \%$ at a threshold of $70-80 \mathrm{pg} / \mathrm{ml})$ yet low specificity $(56.7 \%-60 \%$ at a threshold of $80 \mathrm{pg} / \mathrm{ml})$ in the IC diagnosis [30-32]. Though its sensitivity and specificity may vary due to the difference in the threshold chosen and the performance of the diagnostic kits, the results in this study was still disappointing. At the threshold of $100 \mathrm{pg} / \mathrm{ml}$, only $30 \%$ of the cases were tested positive for BDG within seven days of disease onset. Further investigation during the follow-up showed $29.7 \%$ of the false negative results were due to insufficient increase compared to the threshold, and $14.8 \%$ were due to the lag in increase. Some studies previously suggested to use the negative results of BDG to exclude IC diagnosis and allow discontinuation of the empirical therapy $[30,33,34]$. However, this might delay the preemptive therapy as BDG in this study reacted slowly (averagely 3 days later) and rose insufficiently, which might cause falsenegativity. Using the decrease in BDG levels to evaluate the treatment response, as suggested in previous 
studies [35], is also unreliable, since many factors can cause false positivity in BDG, such as albumin administration [31]. Thus, the results in this study reminded clinicians that the result of BDG, either positive or negative, should not be overly relied on, and the diagnostic culture should be combined to determine the diagnosis. Despite these limitations, ten patients in this study received preemptive therapy according to BDG results, indicating BDG could still respond rapidly and guide timely preemptive therapy in certain circumstances. To further improve diagnostic value, many other markers have been suggested to be combined with BDG, including traditional inflammatory markers (e.g. hsCRP and platelet count) [36] and other fungal antigen (mannan or anti-mannan immunoglobulin $\mathrm{M}$ ) [32].

In this study, the antifungal resistance/non-WT mainly existed in fluconazole (14.4\%) and voriconazole (9.6\%), similar to the worldwide data, in which fluconazole resistance in all Candida spp. ranged from $8.1 \%$ to $39.3 \%$, followed by voriconazole ranging from $5.4 \%$ to $14.0 \%$, Echinocandins ranging from $1.4 \%$ to $2.1 \%$ and Amphotericin $B$ ranging from $0 \%$ to $1 \%[6,7,11,25,26]$. Specifically, fluconazole resistance was prevalent in C.tropicalis (37.0\%) and C.glabrata (24.1\%) in this study, relatively more common than those reported in previous Chinese studies (3.1-11.6\% for C.tropicalis and $12.0-18.5 \%$ for C.glabrata, respectively) $[6,11,13,14,25]$. Voriconazole non-WT C.glabrata isolates in this study (58.6\%) was also more common compared to previous studies $(0-17.8 \%)[2,4,11,14,25,37,38]$. This might indicate a rise in the resistance to azoles in C.glabrata and C.tropicalis in the recent years, which was also observed in the previous studies $[14,25]$. These findings indicate that clinicians should be cautious to use fluconazole/ voriconazole when C.glabrata and C.tropicalis are suspected to be the causative agents. Though the overall resistance to Echinocandins is uncommon, C.glabrata (10.3\%) and C.tropicalis (7.4\%) could develop resistance to Echinocandins in this study. Furthermore, this study found that previous exposure to fluconazole/ voriconazole could mutually increase the risk of developing resistance to voriconazole/ fluconazole. Exposure to caspofungin could also increase the risk of micafungin resistance. Further tests also confirmed the existence of cross-resistance between fluconazole and voriconazole, and between caspofungin and micafungin. All of these indicate the need of performing a drug-susceptibility test no matter the type of antifungal drug, azoles or Echinocandins, is used, especially when the patient has previous antifungal drug exposure. Amphotericin $\mathrm{B}$ is an alternative when multiresistance is evident, since this study together with most previous studies reported non-resistance to Amphotericin B in all the five common Candida species $[6,11,14,26]$.

In terms of treatment, apart from the systematic antifungal therapy, there are two essential interventions contributing to an effective infection control: source control and timely administration of systematic antifungal therapy. In the present study, 16 candidemia patients did not receive any systematic antifungal treatment. Five of them recovered after changing CVC or hemodialysis tubes. One acute abdomen induced candidemic patient recovered after surgery. Eight non-candidemic IC patients recovered after surgery or drainage without systematic antifungal therapy. All of these cases demonstrated the importance of effective source control. Previous studies also demonstrated that failure or delay in source control significantly increased the mortality risk (OR: 6.78-77.40) $[10,26]$. Timely systematic antifungal drug administration is also essential which can significantly reduce mortality [10]. Empirical treatment was strongly recommended in ICU hospitalized patients suspected with IC [39]. In this study, more than 
half of the patients received antifungal treatment within 24 hours, which might contribute to the lower mortality. However, we failed to detect any significant association between prophylaxis/ empirical treatment and better survival. This might be due to insufficient dosage administrated in the prophylaxis/ empirical treatment. $53.5 \%(8 / 15)$ of those who received prophylaxis initiated with fluconazole/ voriconazole $\leq 200 \mathrm{mg} / \mathrm{d}$, while the recommended dosage was $400 \mathrm{mg} / \mathrm{d}$ [39]. Also, 61.9\% (13/21) received empirical therapy with fluconazole/ voriconazole less than the recommended $400 \mathrm{mg} / \mathrm{d}$.

Currently, Echinocandins are recommended as the first-line therapy in both empirical and targeted therapy of IC, and fluconazole is an alternative only recommended in patients with hemodynamic stability and without fluconazole resistance $[1,39]$. However, in this study, the majority of patients received fluconazole as initial therapy and main therapy, even though $28.0 \%$ (59/211) of the isolates had exposure to fluconazole before isolation. After adjustment in the multivariable regression models, initiating antifungal therapy with fluconazole also had a better survival outcome compared to micafungin. This might be due to the small proportion of C.krusei and C.glabrata infections included in this study, and the relatively low resistance/non-WT in the isolates. This indicated that fluconazole could still be a cost-effective choice as long as the resistance was not evident.

The attributable mortality rate of IC could exceed $70 \%$ while the majority reported it to be $10 \%-38 \%[1,3-6]$. Regardless of the evidence of statistical significance, most studies, including the present study, reported lower mortality rates in C.parapsilosis $[1,27]$ and higher mortality rates in C.glabrata $[6,13,27]$. Particularly, one C.parapsilosis infected patient enrolled in this study presented with three episodes of IC with the longest duration of 143 days, indicating the relatively weak virulence of C.parapsilosis. In terms of baseline medical condition, the majority of studies reported advanced age, neutropenia, severe sepsis, respiratory and renal failure, and iatrogenic factors including ICU hospitalization, mechanical ventilation and dialysis were associated with increased mortality $[3,8,13,26,38]$, while appropriate empiric antifungal therapy administrated within five days and proven catheter-related candidemia were found to be protective factors against early mortality [8]. In this study, we also confirmed the CVC induced IC to be a protective factor, and further found hematologic malignancies, digestive tract perforation, heart failure, long-term administration of broad-spectrum antibiotics and TPN to be risk factors for increased mortality. Due to these differences in the baseline conditions of the included population, infected sites and Candida species, time to initiate the therapy and the choice of antifungal drugs, the specific mortality in each study differed a lot. The crude and attributable 30 -day mortality rate in this study was $24.5 \%$ and $18.9 \%$ respectively, lower than most previous studies. This might be due to the large proportion of C.parapsilosis infections, more children patients included, and timely systematic antifungal drug administration in this study. In addition, the median time to death after diagnosis was reported to be 14-15 days [3, 13], which was consistent with this study.

There were several limitations in this study. First, as a retrospective study, many tests investigated were not conducted or monitored regularly, including colonization, 1-3- $\beta$-D-glucan and culture re-examination which might influence the determination of infective condition and bias the analytic results. The mortality outcomes in $13.1 \%(24 / 183)$ of the patients were missing which might underestimate the mortality rate 
as many patients with missing outcomes requested a discharge regardless of a poor prognosis. Second, due to data availability, only IC patients were included. The lack of a control group without invasive candidiasis with similar demographic characteristics limited the ability of this study to explore the true risk factors of developing IC. Third, as a single-center study, the collected data was limited even though the ten-year data was gathered. The small sample size might reduce the power of this study to detect a significance when conducting statistical analysis. Thus, a large-scale multi-center prospective study is required to further investigate the characteristics of invasive candidiasis.

\section{Conclusions}

In conclusion, the incidence of IC had been declining in this center in the recent five years due to the intensified preventive surveillance. Senior people were the major affected population, and colonization was a prevalent risk factor to be aware. C. albicans was still the most common species while C.glabrata infection had the highest mortality. Culture was still the major method for diagnosis. 1-3- $\beta$-D-glucan had limited sensitivity to facilitate diagnosis. Fluconazole resistance/ non-WT was relatively common in this study and was associated with previous exposure to fluconazole or voriconazole. Fluconazole was the most commonly used drug throughout the disease course as initiating therapy with fluconazole was found to be a protective factor against mortality in this study. To reduce the limitations by missing data and small sample size, a multi-center prospective study is needed to further investigate the characteristics of invasive candidiasis.

\section{Declarations}

Funding: This study was supported by Major Infectious Diseases Such as AIDS and Viral Hepatitis Prevention and Control Technology Major Projects (2018ZX10712001-011).

Data availability: Data including the medical records of each case is not available for share.

Materials availability: Materials including clinical Candida isolates are not available for share.

Code availability: Stata code is available upon requested.

Authors' contributions: All the authors involved in the study design. Zhihui Yang collected the clinical data of patients, conducted statistical analyzation, created the initial manuscript and revised the manuscript according to the suggestions of other authors. Yinggai Song involved in the identification and drugsusceptibility test of the isolates. Ruoyu Li helped to revise the manuscript and supervise the whole research project. All authors read and approved the final manuscript.

Conflicts of interest/Competing interests: The authors declare that they have no conflict of interest. 
Ethics approval: This study was approved by the ethics committee of Peking University First Hospital. The need for informed consent was waived by the Clinical Research Ethics Committee as this was a retrospective study.

Consent for publication: All the authors agree to publish and the written consent for publication has been obtained.

Acknowledgements: This study was funded by Major Infectious Diseases Such as AIDS and Viral Hepatitis Prevention and Control Technology Major Projects (2018ZX10712001-011), which were supported by the Ministry of Science and Technology of the People's Republic of China.

\section{References}

1. Pappas PG, Lionakis MS, Arendrup MC, Ostrosky-Zeichner L, Kullberg BJ. Invasive candidiasis. Nat Rev Dis Primers. 2018;4(1); doi:10.1038/nrdp.2018.26.

2. Pfaller MA, Diekema DJ. Epidemiology of invasive candidiasis: a persistent public health problem. Clin Microbiol Rev. 2007;20(1):133-63. doi:10.1128/cmr.00029-06.

3. Guo F, Yang Y, Kang Y, Zang B, Cui W, Qin B et al. Invasive candidiasis in intensive care units in China: a multicentre prospective observational study. J Antimicrob Chemother. 2013;68:1660-1668. doi:10.1093/jac/dkt083.

4. Wisplinghoff H, Ebbers J, Geurtz L, Stefanik D, Major Y, Edmond MB et al. Nosocomial bloodstream infections due to Candida spp. in the USA: species distribution, clinical features and antifungal susceptibilities. Int J Antimicrob Agents. 2014;43(1):78-81. doi:10.1016/j.ijantimicag.2013.09.005.

5. Rodrigues LS, Motta FA, Picharski GL, Vasconcelos TM, Riccieri MC, Dalla-Costa UM. Invasive candidiasis Risk factor for mortality in a pediatric tertiary care hospital in south of Brazil. Medicine (Baltimore). 2019;98(23):e15933-e. doi:10.1097/MD.0000000000015933.

6. Zeng Z, Tian G, Ding Y, Yang K, Deng J, Liu J. Epidemiology, antifungal susceptibility, risk factors and mortality of invasive candidiasis in neonates and children in a tertiary teaching hospital in Southwest China. Mycoses. 2020;63(11):1164-74. doi:10.1111/myc.13146.

7. Wang L, Tong Z, Wang Z, Xu L, Wu Y, Liu Y et al. Single-center retrospective study of the incidence of, and risk factors for, non- $C$. albicans invasive candidiasis in hospitalized patients in China. Med Mycol. 2014;52(2):115-22.

8. Yang Z-T, Wu L, Liu X-Y, Zhou M, Li J, Wu J-Y et al. Epidemiology, species distribution and outcome of nosocomial Candida spp. bloodstream infection in Shanghai. BMC Infect Dis. 2014;14(1):241-250. doi:10.1186/1471-2334-14-241.

9. Tortorano AM, Prigitano A, Lazzarini C, Passera M, Montagna MT. A 1-year prospective survey of candidemia in Italy and changing epidemiology over one decade. Infection. 2013;41(3):655-62.

10. Kollef M, Micek S, Hampton N, Doherty JA, Kumar A. Septic shock attributed to Candida infection: Importance of empiric therapy and source control. Clin Infect Dis. 2012;54(12): 1739-46. 
doi:10.1093/cid/cis305.

11. Liu W, Tan J, Sun J, Xu Z, Li M, Yang Q et al. Invasive candidiasis in intensive care units in China: In vitro antifungal susceptibility in the China-SCAN study. Journal Antimicrob Chemother. 2014;69(1):162-7. doi:10.1093/jac/dkt330.

12. Tan BH, Chakrabarti A, Li RY, Patel AK, Watcharananan SP, Liu Z et al. Incidence and species distribution of candidaemia in Asia: a laboratory-based surveillance study. Clin Microbiol Infect. 2015;21(10):946-53.

13. Li Y, Du M, Chen L-a, Liu Y, Liang Z. Nosocomial bloodstream infection due to Candida spp. in China: species distribution, clinical features, and outcomes. Mycopathologia. 2016;181(7):485-95. doi:10.1007/s11046-016-9997-3.

14. Xu, Ying-Chun, Wang, He, Hsueh, Po-Ren. Epidemiology of candidemia and antifungal susceptibility in invasive Candida species in the Asia-Pacific region. Future Microbiol. 2016;11(11):1461-77. doi:10.2217/fmb-2016-0099.

15. Donnelly JP, Chen SC, Kauffman CA, Steinbach WJ, Baddley JW, Verweij PE et al. Revision and Update of the Consensus Definitions of Invasive Fungal Disease From the European Organization for Research and Treatment of Cancer and the Mycoses Study Group Education and Research Consortium. Clin Infect Dis. 2019;71(6):1367-76. doi:10.1093/cid/ciz1008.

16. Eggimann P, Pittet D. Candida colonization index and subsequent infection in critically ill surgical patients: 20 years later. Intensive Care Med. 2014;40(10):1429-48. doi:10.1007/s00134-014-3355-z.

17. León C, Ruiz-Santana S, Saavedra P, Almirante B, Nolla-Salas J, álvarez-Lerma F et al. A bedside scoring system ("Candida score") for early antifungal treatment in nonneutropenic critically ill patients with Candida colonization. Crit Care Med. 2006;34(3):730-7. doi:10.1097/01.CCM.0000202208.37364.7D.

18. Leroy G, Lambiotte F, Thévenin D, Lemaire C, Leroy O. Evaluation of "Candida score" in critically ill patients: a prospective, multicenter, observational, cohort study. Ann Intensive Care. 2011;1(1):50. doi:10.1186/2110-5820-1-50.

19. Song Y, Chen X, Yan Y, Wan Z, Liu W, Li R. Prevalence and antifungal susceptibility of pathogenic yeasts in China: A 10-year retrospective study in a teaching hospital. Front Microbiol. 2020;11:1401. doi:10.3389/fmicb.2020.01401.

20. StataCorp. Stata Statistical Software: Release 15. College Station, TX: StataCorp LLC.2017.

21. Chiang $\mathrm{CH}$, Pan SC, Yang TS, Matsuda K, Chang SC. Healthcare-associated infections in intensive care units in Taiwan, South Korea, and Japan: recent trends based on national surveillance reports. Antimicrob Resist Infect Control. 2018;7(1):129. https://doi.org/10.1186/s13756-018-0422-1.

22. Yapar N. Epidemiology and risk factors for invasive candidiasis. Ther Clin Risk Manag. 2014;10:95105. doi:http://dx.doi.org/10.2147/TCRM.S40160.

23. Kett DH, Azoulay E, Echeverria PM, Vincent JL. Candida bloodstream infections in intensive care units: analysis of the extended prevalence of infection in intensive care unit study. Crit Care Med. 2011;39(4):665-70. 
24. Pfaller MA, Messer SA, Moet GJ, Jones RN, Castanheira M. Candida bloodstream infections: comparison of species distribution and resistance to echinocandin and azole antifungal agents in Intensive Care Unit (ICU) and non-ICU settings in the SENTRY Antimicrobial Surveillance Program (2008-2009). Int J Antimicrob Agents. 2011;38(1):65-9.

25. Xiao M, Sun ZY, Kang M, Guo DW, Liao K, Chen CA et al. Five-year national surveillance of invasive candidiasis: Species distribution and azole susceptibility from the China Hospital Invasive Fungal Surveillance Net (CHIF-NET) Study. J Clin Microbiol. 2018;56(8):e00577-18. doi:https://doi.org/10.1128/JCM.00577-18.

26. Hsu J-F, Lai M-Y, Lee C-W, Chu S-M, Wu IH, Huang H-R et al. Comparison of the incidence, clinical features and outcomes of invasive candidiasis in children and neonates. BMC Infect Dis. 2018;18(1):194. doi:10.1186/s12879-018-3100-2.

27. Horn David L, Neofytos D, Anaissie Elias J, Fishman Jay A, Steinbach William J, Olyaei Ali J et al. Epidemiology and outcomes of candidemia in 2019 patients: Data from the prospective antifungal therapy alliance registry. Clin Infect Dis. 2009;48(12):1695-703. doi:10.1086/599039.

28. Lau AF, Kabir M, Chen SCA, Playford EG, Marriott DJ, Jones M et al. Candida colonization as a risk marker for invasive candidiasis in mixed medical-surgical intensive care units: Development and evaluation of a simple, standard protocol. J Clin Microbiol. 2015;53(4):1324-30. doi:10.1128/jcm.03239-14.

29. Dubau B, Triboulet $C$, Winnock $S$. Use of the colonization index. Annales françaises d'anesthésie et de réanimation. 2001;20(4):418-20. doi:10.1016/s0750-7658(01)00375-6.

30. Cohen JF, Ouziel A, Matczak S, Brice J, Spijker R, Lortholary $O$ et al. Diagnostic accuracy of serum $(1,3)$-beta-d-glucan for neonatal invasive candidiasis: systematic review and meta-analysis: $(1,3)$ Beta-D-Glucan to detect neonatal invasive candidiasis. Clin Microbiol Infect. 2019; doi:10.1016/j.cmi.2019.09.010.

31. Lo Cascio G, Lo Cascio G, Koncan R, Koncan R, Stringari G, Stringari G et al. Interference of confounding factors on the use of $(1,3)$-beta-D-glucan in the diagnosis of invasive candidiasis in the intensive care unit. Eur J Clin Microbiol Infect Dis. 2015;34(2):357-65. doi:10.1007/s10096-014-2239$z$.

32. Li F, Yu X, Ye L, Zhou G, Wang L, Luo Y. Clinical value of (1,3)- $\beta$-D-glucan, mannan, antimannan IgG and IgM antibodies in diagnosis of invasive candidiasis. Med Mycol. 2019;57(8):976-86. doi:10.1093/mmy/myy158.

33. Ampel NM. Serum 1,3-[beta]-d-Glucan levels predict intra-abdominal candidiasis. NEJM journal watch infectious diseases. 2013. doi:10.1056/nejm-jw.NA32839.

34. De Pascale G, Posteraro B, D'Arrigo S, Spinazzola G, Gaspari R, Bello G et al. (1,3)- $\beta$-d-Glucan-based empirical antifungal interruption in suspected invasive candidiasis: A randomized trial. Crit Care. 2020;24(1):1-10. doi:10.1186/s13054-020-03265-y.

35. Jaijakul S, Vazquez JA, Swanson RN, Ostrosky-Zeichner L. (1,3)- $\beta$-D-Glucan as a prognostic marker of treatment response in invasive candidiasis. Clin Infect Dis. 2012;55(4):521-6. 
doi:10.1093/cid/cis456.

36. Guo J, Wu Y, Lai W, Lu W, Mu X. The diagnostic value of (1,3)-beta-D-glucan alone or combined with traditional inflammatory markers in neonatal invasive candidiasis. BMC Infect Dis. 2019;19(1):716. doi:10.1186/s12879-019-4364-x.

37. Castanheira M, Messer SA, Rhomberg PR, Pfaller MA. Antifungal susceptibility patterns of a global collection of fungal isolates: Results of the SENTRY Antifungal Surveillance Program (2013). Diagn Microbiol Infect Dis. 2016;85(2):200-4. doi:10.1016/j.diagmicrobio.2016.02.009.

38. Chi H-W, Yang Y-S, Shang S-T, Chen K-H, Yeh K-M, Chang F-Y et al. Candida albicans versus nonalbicans bloodstream infections: The comparison of risk factors and outcome. J Microbiol Immunol Infect. 2011;44(5):369-75. doi:10.1016/j.jmii.2010.08.010.

39. Pappas PG, Kauffman CA, Andes DR, Clancy CJ, Marr KA, Ostrosky-Zeichner L et al. Clinical practice guideline for the management of candidiasis: 2016 update by the infectious diseases society of America. Clin Infect Dis. 2016;62(4):409-17. doi:10.1093/cid/civ1194.

\section{Tables}

Due to technical limitations, table 1-4 is only available as a download in the Supplemental Files section.

\section{Figures}


506 yeast isolates cultured from bloodstream or deep-seated fluid/tissue during 2010-2019

480 Candida isolates cultured from bloodstream or deep-seated fluid/tissue
26 non-Candida yeast isolates including:

- Cryptococcus spp. 15 isolates;

- Trichosporon spp. 5 isolates;

- Rhodotorula glutinis 4 isolates;

- Wickerhamomyces/Pichia anomalus 2 isolates.
- 19 isolates were obtained from outpatients;

- 25 isolates were obtained from inpatients with missing information.
436 Candida isolates obtained from hospitalized patients with comprehensive medical records

212 Candida isolates obtained from 183 episodes of proven invasive candidiasis occurred in 177 patients
224 isolates were obtained from non-sterile sties:

- 123 isolates were cultured from bronchoalveolar lavage fluid (BALF);

- 5 isolates were cultured from bronchial secretion;

- 15 isolates were cultured from peritoneal dialysate;

- 4 isolates were cultured from double-J stent / urinary catheter;

- 73 isolates were cultured from pleural / abdominal drainage (placed more than $24 \mathrm{~h}$ ago);

- 2 isolates were cultured from open wounds / tissue (diabetic foot and fistula);

- 2 isolates were clinically cosidered contamination.

\section{Figure 1}

STROBE diagram of study population selection. 
0.45

0.4

0.35

0.3

0.25

0.2

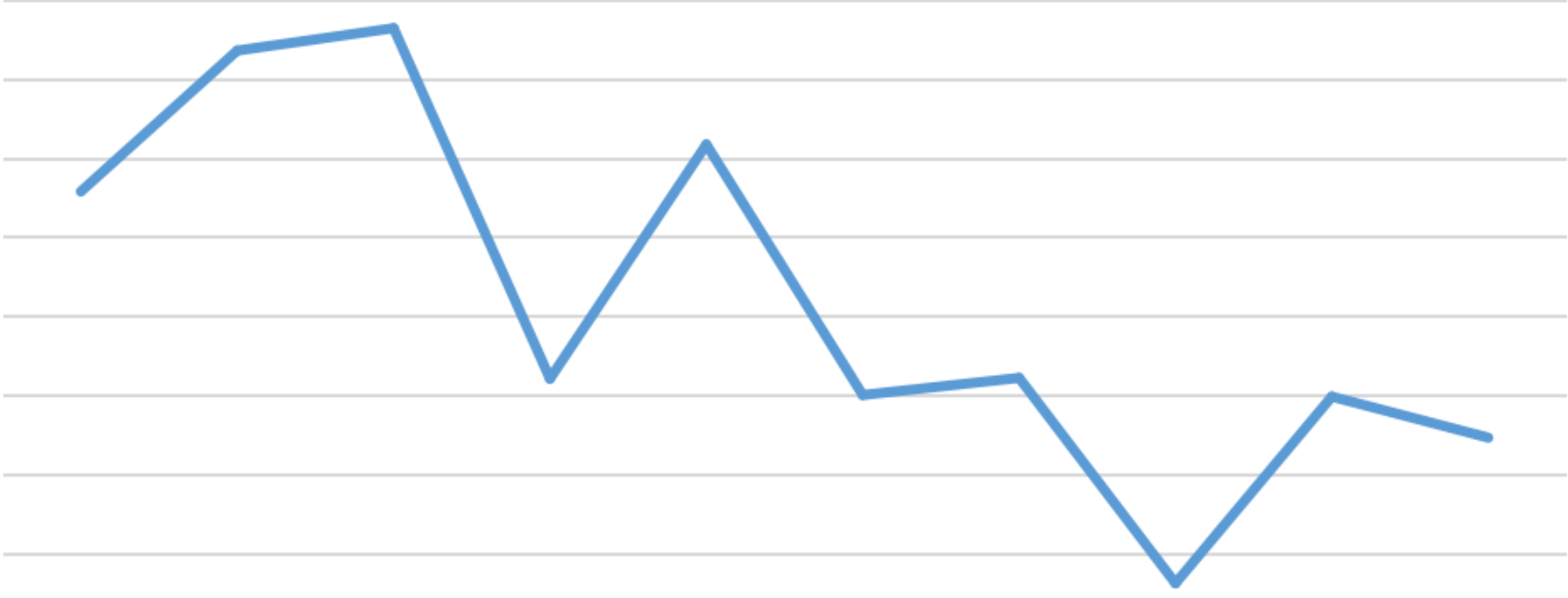

0.05

0

$\begin{array}{llllllllll}2010 & 2011 & 2012 & 2013 & 2014 & 2015 & 2016 & 2017 & 2018 & 2019\end{array}$

\section{Figure 2}

Incidence of invasive candidiasis during 2010-2019.

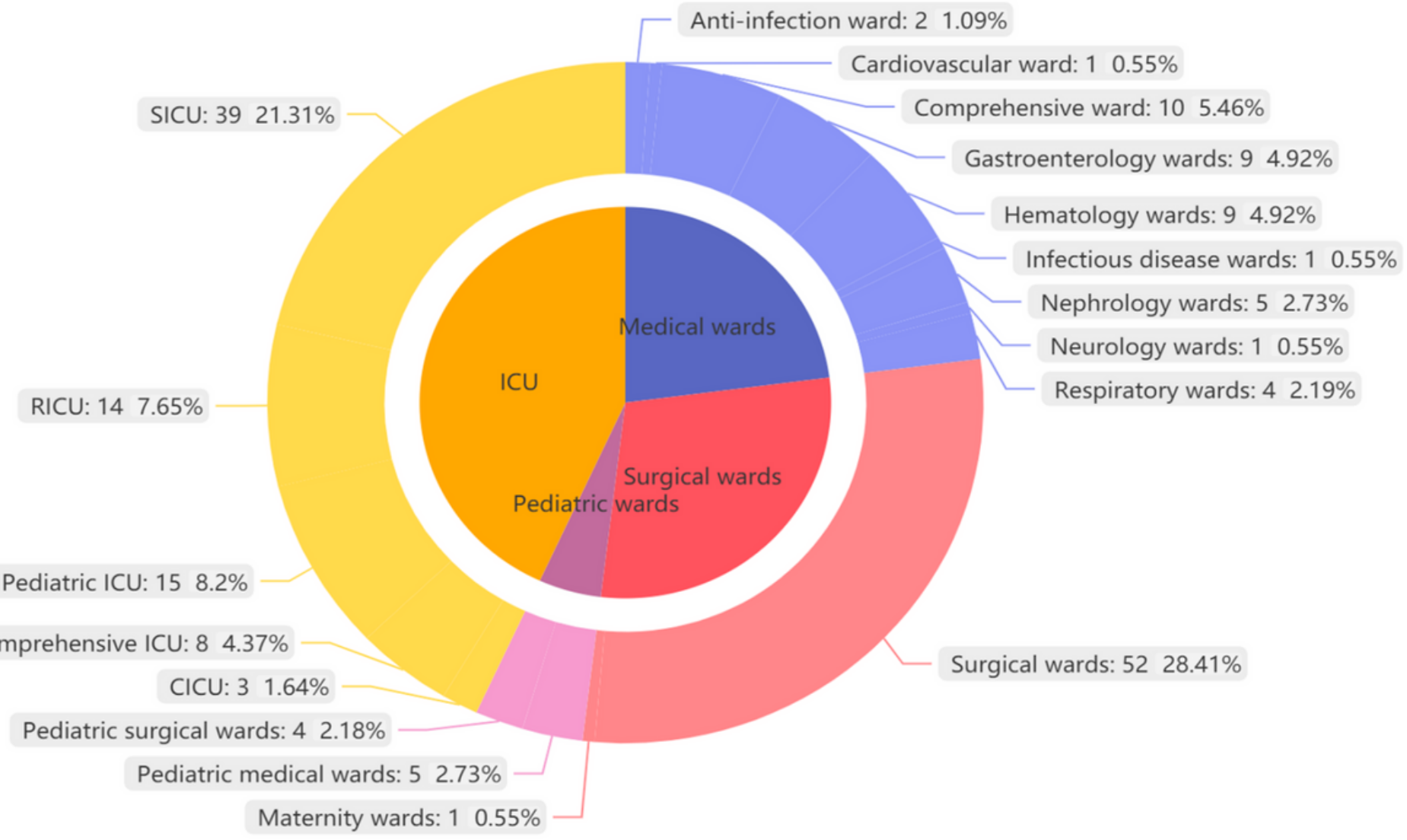

Page 19/21 


\section{Figure 3}

Distribution of invasive candidiasis in different wards. CICU: Cardiac intensive care unit; ICU: Intensive care unit; RICU: Respiratory intensive care unit; SICU: Surgical intensive care unit.

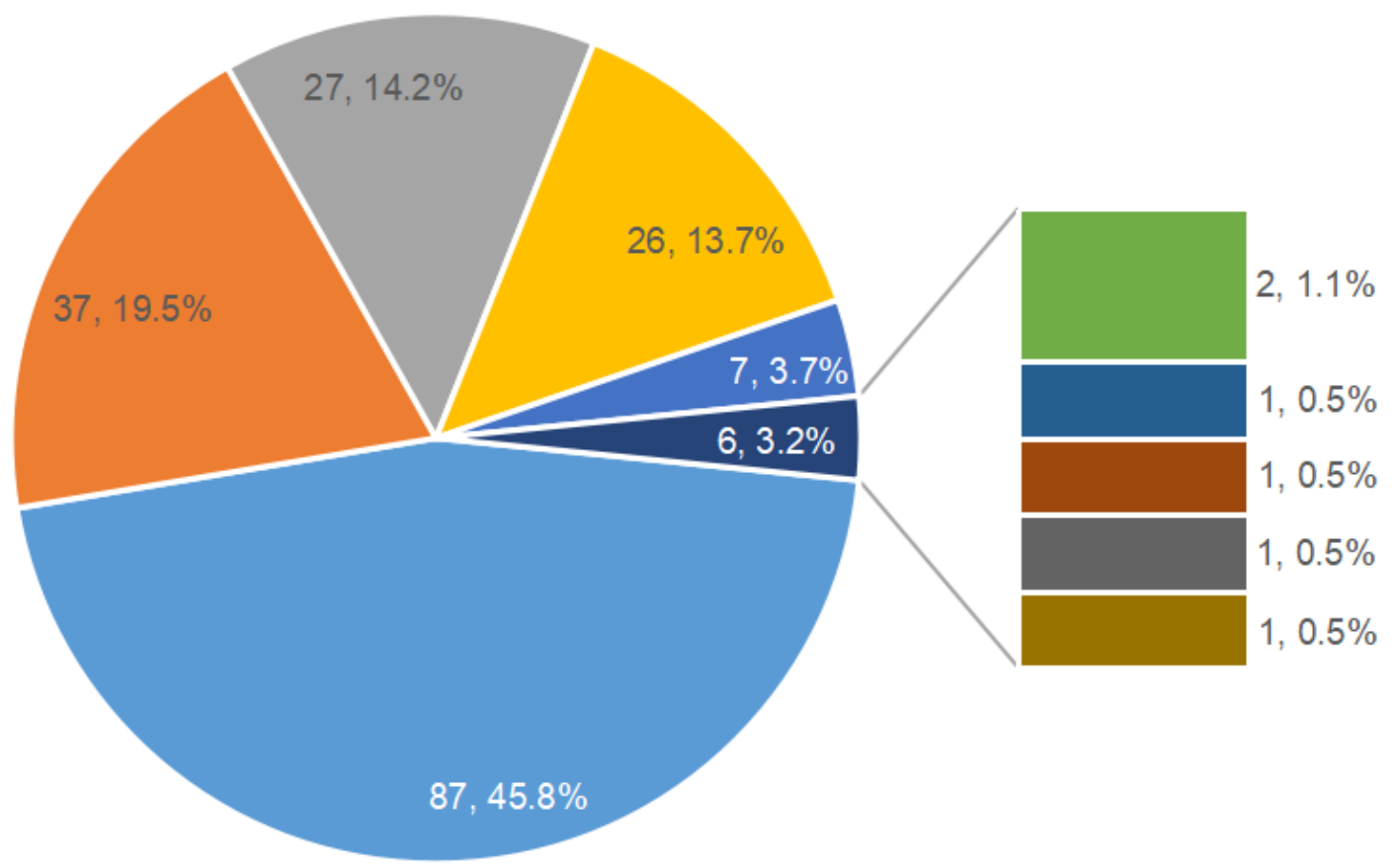

- Candida albicans

- Candida parapsilosis

- Candida glabrata

- Candida tropicalis

- Candida krusei

- Candida inconspicua

- Candida kefyr

- Candida lipolytica

- Candida carpophila

- Candida guilliermondi

\section{Figure 4}

Species distributions of the included the isolates. Note: The total number of the included isolates was 190, since seven episodes were infected by mixed species. Four of them were infected by Candida albicans + Candida glabrata, one by Candida albicans + Candida tropicalis, one by Candida carpophila + Candida guilliermondi and one by Candida krusei + Candida tropicalis. 


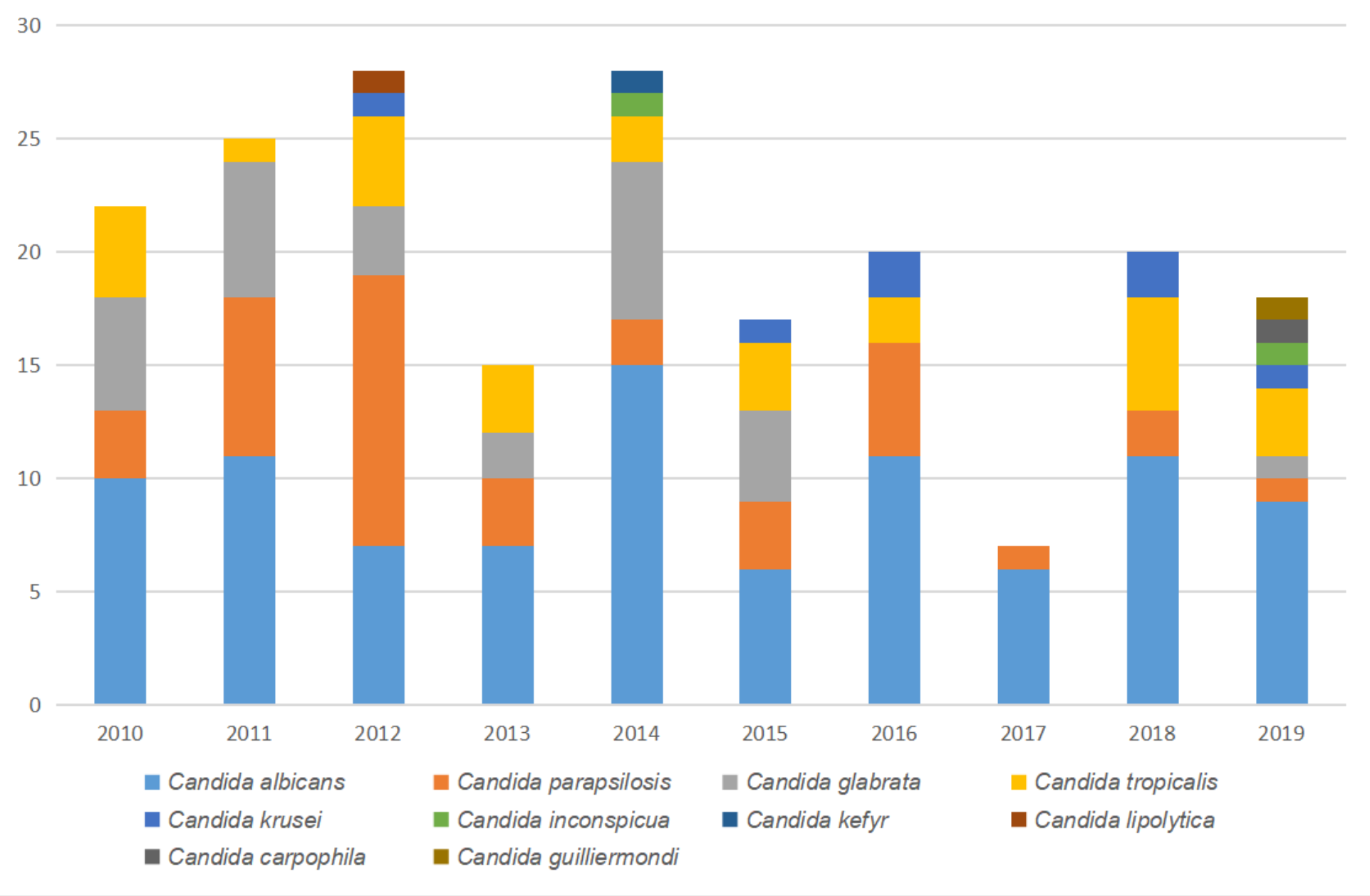

\section{Figure 5}

Species distribution of Candida isolates during 2010-2019.

\section{Supplementary Files}

This is a list of supplementary files associated with this preprint. Click to download.

- supplementarytables2.docx

- Tables.pdf 